\title{
VARIATION OF CONFORMAL SPHERES BY SIMULTANEOUS SEWING ALONG SEVERAL ARCS( $\left.{ }^{\mathbf{1}}\right)$
}

\author{
BY
}

T. L. McCOY

\begin{abstract}
Let $M$ be a closed Riemann surface of genus zero, $\Gamma$ a tree on $M$ with branches $\Gamma_{j}$, and $p_{0}$ a point of $M-\Gamma$. A family of neighboring topological surfaces $M(\varepsilon)$ is formed by regarding each $\Gamma_{j}$ as a slit with edges $\Gamma_{j}^{-}$and $\Gamma_{j}^{+}$, and re-identifying $p$ on $\Gamma^{-}$with $p+\varepsilon \chi_{j}(p, \varepsilon)$ on $\Gamma_{j}^{+}$, with $\chi_{j}$ vanishing at the endpoints of $\Gamma_{j}$. We assume the $\Gamma_{j}$ and $\chi_{j}$ are such that, under a certain natural choice of uniformizers, the $M(\varepsilon)$ are closed Riemann surfaces of genus zero. Then there exists a unique function $f\left(p, \varepsilon ; p_{0}\right)$ mapping $M(\varepsilon)$ conformally onto the complex number sphere, with normalization $f\left(p_{0}, \varepsilon ; p_{0}\right)=0, f^{\prime}\left(p_{0}, \varepsilon ; p_{0}\right)=1$. Under appropriate smoothness hypotheses on $\Gamma$ and the $\chi_{j}$, we find the first variation of $f$ as a function of $\varepsilon$. Further, we obtain smoothness results for $f$ as a function of $\varepsilon$. The problem is connected with the study of the extremal schlicht functions; that is, the schlicht mappings of the unit disc corresponding to boundary points of the coefficient bodies.
\end{abstract}

1. Introduction. Let $M$ be a Riemann surface and to simplify the exposition we assume at the outset that $M$ is closed and of genus zero. Let us briefly describe a method which has been used ([4, pp. $23 \mathrm{ff}]$, and [5, p. 310]) to deform $M$ into a neighboring topologically equivalent Riemann surface. We let $\Gamma$ be an analytic arc on $M$, lying within a parametric disc, and suppose there is a function

$$
\varphi(p, \varepsilon)=p+\varepsilon \chi(p, \varepsilon)
$$

defined on $\Gamma$ and mapping $\Gamma$ one-to-one onto an arc $\tilde{\Gamma}(\varepsilon)$ having the same endpoints; let us assume for simplicity that $\tilde{\Gamma}(\varepsilon)$ does not cross $\Gamma$. Now regard $\Gamma$ and $\tilde{\Gamma}(\varepsilon)$ as having two edges, remove the interior $R(\varepsilon)$ of the region with boundary $\Gamma^{+}(\varepsilon) \cup \tilde{\Gamma}^{-}(\varepsilon)$, then sew $\tilde{\Gamma}^{+}(\varepsilon)$ to $\Gamma^{-}(\varepsilon)$ in such a way that the point $p$ on $\Gamma^{-}$is joined to $\varphi(p, \varepsilon)$ on $\tilde{\Gamma}^{+}(\varepsilon)$. The result is a surface, $M(\varepsilon)$, topologically equivalent to $M$. We make $M(\varepsilon)$ into a Riemann surface by introducing a new set of local parameters as described in the following paragraph.

Presented to the Society, January 17, 1972 under the title $A$ surface variation for extremal schlicht functions; received by the editors November 18, 1975.

AMS (MOS) subject classifications (1970). Primary 30A30.

(1) A significant portion of the research leading to this paper was carried out at the University of Glasgow, 1970-1971, where the author spent a sabbatical year.

C American Mathematical Society 1977 
For $p$ on $M-R(\varepsilon)$, we regard $p$ as a point of $M$ and of $M(\varepsilon)$ both, and use the same local parameters on both surfaces for such $p$. For $p$ on $M(\varepsilon)$ and lying over points $p_{0} \in \Gamma^{-}, \varphi\left(p_{0}, \varepsilon\right)$ on $\tilde{\Gamma}^{+}(\varepsilon)$, a parametric disc is to consist of a half-neighborhood $N$ of $p_{0}$ and the half-neighborhood $\varphi(N, \varepsilon)$ of $\varphi\left(p_{0}, \varepsilon\right)$ chosen so that neither set intersects an endpoint of $\Gamma$. If $\tau(p)$ is the local uniformizer on $M$, the uniformizer on $M(\varepsilon)$ is $\tilde{\tau}$;

$$
\tilde{\tau}(p)= \begin{cases}\tau(p), & p \in N, \\ \tau\left(\varphi^{-1}(p, \varepsilon)\right), & p \in \varphi(N, \varepsilon) .\end{cases}
$$

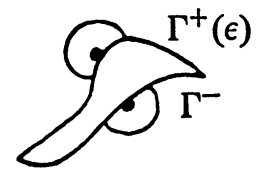

These local parameters make a Riemann surface out of the surface $M^{\prime}(\varepsilon)$, consisting of $M(\varepsilon)$ with the endpoints of $\Gamma$ removed. $M^{\prime}(\varepsilon)$ is conformally equivalent to the sphere with two slits removed (cf. [6, p. 224]), and under appropriate conditions on $\varphi$, these two slits reduce to points. $M(\varepsilon)$ is then conformally equivalent to the sphere, and this conformal mapping induces local uniformizers at the endpoints of $\Gamma$ which make all of $M(\varepsilon)$ a closed surface of genus zero.

We refer the reader to $([1, \mathrm{pp} .69 \mathrm{ff}])$ for a discussion of the case where $M$ is the number sphere, $C$, and $\Gamma$ is a single arc. The proof of the main theorem of that section (called the Sewing Theorem) suggests many of the techniques we use in this paper.

One can proceed to study the variation of functionals as we pass from $M$ to $M(\varepsilon)$. In particular, we will be interested in the function $f\left(p, \varepsilon ; p_{0}\right)$ mapping $M(\varepsilon)$ conformally to the number sphere with $p_{0}$ going to the origin, and $f^{\prime}\left(p_{0}, \varepsilon ; p_{0}\right)=1$ in terms of some particular uniformizer at $p_{0}, p_{0}$ off $\Gamma$. (It should be remarked that, since we can regard $M$ and $M(\varepsilon)$ as having the exterior of $R$ as a common domain with the same system of local parameters, we will frequently use the same notation for points on both surfaces.)

Schiffer and Spencer [5] consider very general variations of the kind described above, for surfaces which are of arbitrary genus. We restrict our attention to conformal spheres. Further, our deformations will be degenerate in the sense that $p \rightarrow \varphi(p, \varepsilon)$ maps $\Gamma$ to itself; let us call this type of deformation a variation by sewing. However, we generalize the problem in a different direction. We let $\Gamma$ be a tree, consisting of disjoint analytic arcs $\Gamma_{j}$, the $\Gamma_{j}$ forming the branches. We define

$$
\varphi(p, \varepsilon)=\varphi_{j}(p, \varepsilon), \quad p \in \Gamma_{j},
$$


$\varphi_{j}(p, \varepsilon)$ a mapping of $\Gamma_{j}$ to itself preserving the endpoints, of the form

$$
\varphi_{j}(p, \varepsilon)=p+\varepsilon \chi_{j}(p, \varepsilon) .
$$

It is to be observed that we cannot choose the $\chi_{j}$ independently of each other and obtain a new surface $M(\varepsilon)$ which is a conformal sphere, for a conformal equivalence between $M$ and $M(\varepsilon)$ will induce local uniformizers at the endpoints of the arcs $\Gamma_{j}$, which will determine the $\chi_{j}$ in terms of analytic continuation from a given $\chi_{k}$. However, it makes perfectly good sense to ask for variational formulae for $f\left(p, \varepsilon ; p_{0}\right)$, given that the sewing deformation can be carried out. Under suitable conditions on the $\chi_{j}$, made explicit in our next section, we will obtain a variational formula $(6.1)$ for $f\left(p, \varepsilon ; p_{0}\right)$. The simpler formula ((6.2), (6.3)) is available if $f\left(p, \varepsilon ; p_{0}\right)$ has its pole at a fixed vertex of $\Gamma$. In a later section, we prove an analyticity property of the first variation of $f$ (Theorem 7.1). We also mention that in applications to specific deformation problems, it is relatively easy to give an analytic description of the sewing on the interior of $\Gamma_{j}$, in terms of the local uniformizers, by writing down $\chi_{j}(p, \varepsilon)$, but difficult to write down formulae describing what happens at the endpoints. Thus we develop the variational formulae in terms of what happens on the interiors of the arcs.

We conclude this introductory section by describing a family of surfaces which are connected by sewing deformations, and which motivated the present study. Schaeffer and Spencer proved [4] that the extremal schlicht functions can be characterized as conformal mappings into the number sphere of surfaces formed as follows: let the points of $M$ be the unit disc, together with $n$ pairs $I_{j}, I_{j}^{\prime}$ of arcs of the unit circumference identified in opposite directions, with further identifications made among the endpoints in such a way as to form a topological sphere, the unit circumference forming a tree on that sphere. The interiors of the arcs are to be identified metrically by a given function $q$, according to the rule

$$
\int_{z_{j}^{\prime}}^{z^{\prime}} q(t) d t=\int_{z_{j}}^{z} q(t) d t
$$

$z_{j}, z_{j}^{\prime}$ identified endpoints of $I_{j}, I_{j}^{\prime}$, and $z \in I_{j}, z^{\prime} \in I_{j}^{\prime}$. (For further details, see [4, pp. $112 \mathrm{ff}]$.) If we vary $q$ in such a way that the endpoints of the pairs $I_{j}, I_{j}^{\prime}$ are preserved, we have a new surface obtained from the old by a process of simultaneous sewing along several arcs; the metric identification of interiors of branches of the tree is the only change. In a later paper $\left({ }^{2}\right)$, we apply our technique in detail to these surfaces, obtaining a variational formula which connects two neighboring extremal schlicht functions, and leads to a family of analytic curves on the boundary of the coefficient body.

$\left.{ }^{2}\right)$ A partial surface variation for extremal schlicht functions, to appear in this journal. 
ADDED IN PROOF. In Lemma 7.1 and Theorem 7.1, also the last sentence of Section 1, read "infinitely differentiable" for "analytic".

It no longer seems clear to the author that the method of Lemma 7.1 yields analyticity.

2. Terminology, and a statement of our basic assumptions. In order to avoid rather cumbersome statements of lemmas and theorems, we set down here the basic hypotheses which we will be assuming throughout.

$M$ is a conformal sphere, $\Gamma$ a tree on $M$. We take $\Gamma=\cup \Gamma_{j}$, where each $\Gamma_{j}$ is analytic in the interior and Hölder-continuous, in terms of a local uniformizer, at the endpoints. Let $a_{j, 1}, a_{j, 2}$ denote the endpoints of $\Gamma_{j}$. We suppose there is a parametric patch $D_{j}$ containing the interior of $\Gamma_{j}$, and an angular region (in terms of local uniformizers) meeting the endpoints of $\Gamma_{j}$.

Let us notice that, by considering $\Gamma$ as the intersection of a sequence of closed curves in $M$, we can speak without ambiguity of a left edge, $\Gamma_{j}^{-}$, and a right edge, $\Gamma_{j}^{+}$, of $\Gamma_{j}$.

The re-identification of edges of $\Gamma_{j}$ is described by $p \rightarrow \varphi_{j}(p, \varepsilon), p \in \Gamma_{j}^{-}$, $\varphi_{j}(p, \varepsilon) \in \Gamma_{j}^{+}$. Let $\tau$ be a local parameter on $\Gamma_{j}$, and supose $\varphi_{j}$ is described in terms of $\tau$ by

$$
\tau \rightarrow \tau+\varepsilon r_{j}(\tau, \varepsilon)
$$

By our hypothesis, $r_{j}$ vanishes at $\tau\left(a_{j, 1}\right)$ and $\tau\left(a_{j, 2}\right)$. We suppose that in the domain $D_{j}, r_{j}(\tau, \varepsilon)$ is analytic in $\tau$, and continuous in $\varepsilon$ for $|\varepsilon| \leqslant \varepsilon_{0}$, and that $r_{j}(\tau, \varepsilon)$ is bounded, independently of $\varepsilon$, on the closure of $\Gamma_{j}$. At the endpoints, $r_{j}$ is to satisfy a Hölder-condition

$$
\left|r_{j}(\tau, \varepsilon)\right|<A\left|\tau-\tau\left(a_{j, k}\right)\right|^{\mu}, \quad k=1,2, \mu>0,
$$

as $p$ tends to $a_{j, k}$ within $D_{j}$, with $A$ independent of $\varepsilon$. Further, $\Gamma_{j}$ is to have a tangent line at both endpoints. We note that the conditions imposed on the representative $r$ of the sewing deformation are invariant under changes of local parameter.

We take $\varphi=\varphi_{j}$ and $r=r_{j}$ for $p \in \Gamma_{j}$, so that we may write the re-identification as one equation,

$$
p \rightarrow \varphi(p, \varepsilon), \quad p \in \Gamma^{-} .
$$
if

Definition 2.1. We say $\Phi(p, \varepsilon)$ is a sewing mapping for the system $\{M, \Gamma, \varphi\}$

(a) $\tilde{p}=\Phi(p, \varepsilon)$ maps $M-\Gamma$ conformally onto a region $M-\Gamma(\varepsilon)$, where $\Gamma(\varepsilon)$ is of the form

$$
\Gamma(\varepsilon)=\bigcup_{j=1}^{n} \Gamma_{j}(\varepsilon),
$$

each $\Gamma_{j}(\varepsilon)$ analytic on its interior and Hölder-continuous out to the 
endpoints. The $\Gamma_{j}(\varepsilon)$ are disjoint except perhaps at endpoints.

(b) $\Phi(p, \varepsilon)$ is continuous onto both edges of $\Gamma_{j}$, and brings points on $\Gamma_{j}^{-}$, $\Gamma_{j}^{+}$identified by $\varphi_{j}(p, \varepsilon)$ onto the same point of $\Gamma_{j}(\varepsilon)$.

Definition 2.2. $M(\varepsilon)$ is the Riemann surface formed by the re-identification of edges of the $\Gamma_{j}$.

We introduce, finally, certain normalized mappings to be used in the sequel. Let us fix $p_{0}$ on $M$, off $\Gamma$, so that $p_{0}$ can be regarded as a point of $M(\varepsilon)$ also. Let $\tau(p)$ be some particular local uniformizer about $p_{0}$. Then, provided the following exist, $\Phi\left(p, \varepsilon ; p_{0}\right)$ is the sewing mapping for the system $\{M, \Gamma, \varphi\}$ which carries $p_{0}$ to $p_{0}$ and has in terms of the parameter $\tau$ about $p_{0}$ an expansion $\tau+c \tau^{3}+\ldots$, for some $c . f\left(p, \varepsilon ; p_{0}\right)$ is the conformal mapping of $M(\varepsilon)$ to the number sphere $C$ having in terms of the parameter $\tau$ about $p_{0}$ an expansion $\tau \oplus a_{2} \tau^{2}+\ldots$ We take $f\left(p ; p_{0}\right)=f\left(p, 0 ; p_{0}\right)$. We define $H\left(p, p_{0}\right)$ as the function mapping $M$ conformally onto $C$, having in terms of $\tau$ about $p_{0}$ an expansion $1 / \tau+a \tau+\ldots$ By the Uniformization Principle ([6, p. 227]), $H\left(p, p_{0}\right)$ exists and is unique. (We are to understand, of course, that the coefficients $a, a_{2}$, and $c$ in the above normalized expansions are nonvanishing coefficients.)

3. Existence questions and an induced sewing problem on $C$. In this section, $C$ always denotes the complex number sphere.

Lemma 3.1. Suppose there is a sewing mapping $\Phi$ for the system $\{C, \Gamma, \varphi\}$. Then there is exactly one sewing mapping

$$
\begin{aligned}
& \Phi(w, \varepsilon ; \infty) \quad \text { with the normalization } \\
& \Phi(w, \varepsilon ; \infty)=w+c(\varepsilon) / w+\cdots, \quad|w|>r .
\end{aligned}
$$

Proof. Suppose there were another such normalized sewing mapping, $\psi$. Then we see easily, by series manipulations, that $\Phi \psi^{-1}$ is a self-map of $C$, having at infinity a simple pole with residue 1 , and zero translation, thus $\Phi \psi^{-1}$ is just the identity, and $\Phi=\psi$.

If there exists a sewing mapping $\Phi$, it is easy to show that we can follow $\Phi$ by an appropriate bilinear mapping to achieve a sewing map with the required normalization.

We remark that, since $1 / w$ is a uniformizer at $\infty$ on $C$, the function $\Phi(w, \varepsilon ; \infty)$ of the above lemma is precisely $\Phi\left(p, \varepsilon ; p_{0}\right)$ when $M$ is $C$ and $p_{0}$ is $\infty$.

LEMMA 3.2. If there is a sewing mapping $\Phi$ for the system $\{M, \Gamma, \varphi\}$, there is precisely one normalized sewing mapping $\Phi\left(p, \varepsilon ; p_{0}\right)$.

Proof. Using the function $H\left(p, p_{0}\right)$, the sewing problem $\{M, \Gamma, \varphi\}$ induces a sewing problem $\{C, \Delta, \psi\}$ on $C$. $\Phi$ exists if and only if a sewing mapping $\psi$ 
exists for the system $\{C, \Delta, \psi\}$. The normalized mapping $\psi_{1}=\psi(w, \varepsilon ; \infty)$ exists uniquely by the preceding lemma, and $H^{-1} \psi_{1} H$ is a normalized sewing mapping for $\{M, \Gamma, \varphi\}$. It is easy to see that if there were two normalized sewing mappings for the system $\{M, \Gamma, \varphi\}$, there would be two normalized sewing mappings for the system $\{C, \Delta, \psi\}$.

Checking that the expansions are correct involves tedious but straightforward series manipulations.

LEMMA 3.3. $M(\varepsilon)$ is conformally equivalent to the sphere if and only if there is a sewing mapping for the system $\{M, \Gamma, \varphi\}$.

Proof. Suppose there is a conformal mapping $f_{\varepsilon}$ of $M(\varepsilon)$ to the number sphere, and let $f_{0}$ map $M$ to the number sphere. Clearly $f_{0}^{-1} \circ f_{e}$ is a sewing mapping for the system $\{M, \Gamma, \varphi\}$.

Suppose a sewing mapping $\Phi$ exists. Then $\Phi^{-1}$ maps $M$ conformally to $M(\varepsilon)$, carrying $\Gamma(\varepsilon)$ to $\Gamma$, with $\Gamma_{j}(\varepsilon)$ corresponding to $\Gamma_{j}$, and endpoints going to endpoints. The mapping $\Phi^{-1}$ induces a system of local parameters on each $\Gamma_{j}$, and to show the image of $M$ under $\Phi^{-1}$ is really $M(\varepsilon)$, we just have to check that this new system of local parameters coincides with the local parameters we previously defined on interior points of the $\Gamma_{j}$. But $\Phi^{-1}$ carries $\tilde{p}$ on $\Gamma_{j}(\varepsilon)$ to points $p, \varphi(p, \varepsilon)$ on $\Gamma_{j}^{-}$and $\Gamma_{j}^{+}$, and since $\varphi(p, \varepsilon)$ is analytic on interior arcs, the system of local parameters induced by $\Phi^{-1}$ on $\Gamma_{j}$ is indeed the same. Since $M(\varepsilon)$ is conformally equivalent to $M$, both are conformally equivalent to $C$.

The relation

$$
f\left(p, \varepsilon ; p_{0}\right)=f\left(\Phi\left(p, \varepsilon ; p_{0}\right), 0, p_{0}\right)
$$

follows by considering the series developments in terms of the special parameter $\tau$ about $p_{0}$.

4. The sewing mapping on the sphere. In this section, we take $M=C$, the sphere of complex numbers $w$, and let $\Gamma$ be a tree on $\Gamma$, not meeting $\Gamma$. Since $w$ itself is a local parameter, we take the sewing on $\Gamma_{j}$ to be $w \rightarrow w+\varepsilon \chi(w, \varepsilon)$, $w$ on $\Gamma^{-}$. For simplicity in notation, let $\Phi_{0}(w, \varepsilon)$ be the normalized sewing mapping $\Phi(w, \varepsilon ; \infty)$ of Lemma 3.1.

We study, in the two lemmas below, the behavior of $\Phi$ as a function of $\varepsilon$. This material is very similar to the work leading to Schaeffer and Spencer's basic variational formula ([4, pp. $23 \mathrm{ff}])$, although of course we do not need to prove the existence of $\Phi$, we are assuming it. In spite of the similarity, we present our arguments in detail because they are slightly different from [4] and because we will need to refer back to certain details later.

Lemma 4.1. Let $\Phi_{0}(w, \varepsilon)$ exist. Then $\Phi_{0}$ satisfies, off $\Gamma$, the functional equation 


$$
\Phi_{0}(w, \varepsilon)=w-\frac{\varepsilon}{2 \pi i} \int_{\Gamma^{-}} \frac{\chi(x, \varepsilon) \Phi_{0}^{\prime}(x, \varepsilon)}{\Phi_{0}(x, \varepsilon)-\Phi_{0}(w, \varepsilon)} d x .
$$

This equation remains valid on $\Gamma^{+}$, including endpoints of the arcs $\Gamma_{j}$, where we regard each $\Gamma_{j}$ as double-edged.

Proof. We write $\omega=\Phi_{0}(w, \varepsilon)$. Each $\Gamma_{j}$ maps to an arc $\Gamma_{j}(\varepsilon)$, and $\Phi^{-1}(\omega, \varepsilon)$ has a jump, say $\varepsilon \lambda_{j}(\omega, \varepsilon)$ as we pass from $\Gamma_{j}^{-}(\varepsilon)$ to $\Gamma_{j}^{+}(\varepsilon)$. Formally, the integral

$$
\frac{\varepsilon}{2 \pi i} \int_{\Gamma_{j}^{-}(\varepsilon)} \frac{\lambda_{j}(u, \varepsilon)}{u-\omega} d u
$$

has precisely the same jump across $\Gamma_{j}(\varepsilon)([2, \mathrm{pp} .42 \mathrm{ff}])$ thus the function

$$
\Phi_{0}^{-1}(\omega, \varepsilon)-\frac{\varepsilon}{2 \pi i} \int_{\Gamma_{j}^{-}(\varepsilon)} \frac{\lambda_{j}(u, \varepsilon)}{u-\omega} d u
$$

is continuous across $\Gamma_{j}(\varepsilon)$. Replacing $\Phi^{-1}(\omega, \varepsilon)$ by $w$, and adding over the $\Gamma_{j}$, $j=1, \ldots, n$, we are led to

$$
w=\Omega(\omega, \varepsilon)+\frac{\varepsilon}{2 \pi i} \int_{\Gamma^{-}(\varepsilon)} \frac{\lambda(u, \varepsilon)}{u-\omega} d u,
$$

with $\lambda=\lambda_{j}$ on $\Gamma_{j}(\varepsilon)$, and $\Omega$ a function regular and vanishing at $\infty$ and having the endpoints of the $\Gamma_{j}(\varepsilon)$ as the only possible singularities. Changing the variable of integration in the above equation, we have

$$
\Omega(\omega, \varepsilon)=w-\frac{\varepsilon}{2 \pi i} \int_{\Gamma^{-}} \frac{\chi(x, \varepsilon) \Phi_{0}^{\prime}(x, \varepsilon)}{\Phi_{0}(x, \varepsilon)-\Phi_{0}(w, \varepsilon)} d x .
$$

(4.3) is valid provided the integral on the right-hand side exists. To settle this question, let us concentrate on the integral

$$
\int_{\Gamma_{j}^{-}} \frac{\chi(x, \varepsilon) \Phi_{0}^{\prime}(x, \varepsilon)}{\Phi_{0}(x, \varepsilon)-\Phi_{0}(w, \varepsilon)} d x
$$

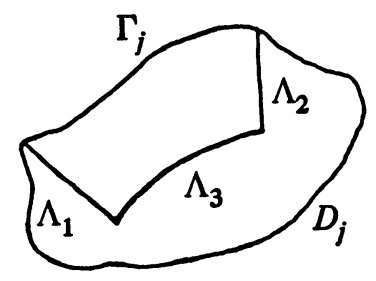

FIGURE 4.1

Because $\Phi_{0}$ is analytic off $\Gamma_{j}$, we can replace the path of integration $\Gamma_{j}^{-}$by $\Lambda_{1} \cup \Lambda_{2} \cup \Lambda_{3}=\Lambda$, lying below $\Gamma_{j}^{-}, \Lambda_{1}, \Lambda_{2}$ line segments of length $s$ making 
positive angles with $\Gamma_{j}^{-}$at the endpoints, and $\Lambda_{3}$ lying a fixed distance $d$ from $\Gamma_{j}$, connecting the free ends of $\Lambda_{1}, \Lambda_{2}$. (See Figure 4.1.) We can suppose $\Lambda$ lies in the domain $D_{j}$ (see $\$ 2$ ).

Let $\rho(x)$ be the distance from $x$ to $\Gamma$. Because $\Phi_{0}$ is a conformal mapping the One-Quarter Theorem [3, p. 214] gives us the estimate

$$
\left|\Phi_{0}^{\prime}(x, \varepsilon) /\left(\Phi_{0}(x, \varepsilon)-\Phi_{0}(w, \varepsilon)\right)\right| \leqslant 4 / \rho(x),
$$

for $|w-x|=\rho(x)$, and because of the mapping properties of $\Phi$, (4.4) holds for $|w-x| \geqslant \rho(x)$. We can now estimate the integrals over the $\Lambda_{n}$. Let us assume $w$ lies outside the region obtained by covering $\Lambda$ by discs of radius $\rho(x)$, and outside the region bounded by $\Gamma_{j}^{-}$and $\Lambda$. We have then

$$
\left|\int_{\Lambda_{3}} \frac{\chi_{j}(x, \varepsilon) \Phi_{0}^{\prime}(x, \varepsilon)}{\Phi_{0}(x, \varepsilon)-\Phi_{0}(w, \varepsilon)} d x\right|<4 M L_{3} / d
$$

where $L_{3}$ is the length of $\Lambda_{3}$ and $M$ is an upper bound for $\left|\chi_{j}(x, \varepsilon)\right|$ on $\Gamma_{j}$. For the integrals over $\Lambda_{1}$ and $\Lambda_{2}$ we have the estimate

$$
\left|\int_{\Lambda_{k}} \frac{\chi_{j}(x, \varepsilon) \Phi_{0}^{\prime}(x, \varepsilon)}{\Phi_{0}(x, \varepsilon)-\Phi_{0}(w, \varepsilon)} d x\right|<4 \int_{\Lambda_{k}} \frac{\chi_{j}(x, \varepsilon)}{\rho(x)} d x, \quad k=1,2
$$

Because of the Hölder condition on $\chi_{j}$ at the endpoints, and the fact that $\rho(x)>C\left|x-a_{k, j}\right|$ for some $C$ determined only by the geometry, we have

$$
\begin{aligned}
\left|\int_{\Lambda_{k}} \frac{\chi_{j}(x, \varepsilon) \Phi_{0}^{\prime}(x, \varepsilon)}{\Phi_{0}(x, \varepsilon)-\Phi_{0}(w, \varepsilon)} d x\right| & <\frac{4 A}{C} \int_{\Lambda_{k}}\left|x-a_{k_{j}}\right|^{\mu-1}|d x| \\
& =\frac{4 A}{C} \int_{0}^{s} r^{\mu-1} d r, \quad k=1,2,
\end{aligned}
$$

this last being a convergent integral, independent of $\varepsilon$.

We have just shown that the integral on the right-hand side of (4.3) is uniformly bounded independently of $\varepsilon$, for $w$ in any region whose closure does not meet $\Gamma^{-}$, where we are thinking of $\Gamma$ as a double-edged curve. Observe that in particular the right-hand side is bounded when $w$ is an endpoint of an arc $\Gamma_{j}$. Now since $\Omega(\omega, \varepsilon)$ is single-valued, and has the endpoints of the $\Gamma_{j}(\varepsilon)$ as the only possible singularities, we can show these singularities are removable if we can show that $\Omega(\omega(w, \varepsilon), \varepsilon)$ is bounded in a full neighborhood of the endpoints of the $\Gamma_{j}$. This is certainly true, as shown above, if $w$ lies outside the domain bounded by $\Gamma_{j}^{-}$and $\Lambda$. If $w$ is in the interior of the domain enclosed by $\Gamma_{j}^{-}$and $\Lambda$, then the integrals over $\Gamma_{j}^{-}$and $\Lambda$ differ by the residue of the integrand at $w$, which is $\chi_{j}(w, \varepsilon)$, uniformly bounded. If $w$ lies a fixed distance from $\Lambda$, the integral 


$$
\int_{\Lambda} \frac{\chi_{j}(x, \varepsilon) \Phi_{0}^{\prime}(x, \varepsilon)}{\Phi_{0}(x, \varepsilon)-\Phi_{0}(w, \varepsilon)} d x
$$

is still bounded, and for $w$ too close to our fixed $\Lambda$, we can simply change $\Lambda$ to get an appropriate bound for the integral, just repeating the argument of the preceding paragraph. $\Omega(\omega(w, \varepsilon), \varepsilon)$ is bounded everywhere off $\Gamma^{-}$, and by continuity is bounded on $\Gamma^{-}$as well.

We know now that $\Omega(\omega, \varepsilon)$ has no singularities in the finite plane. Referring to (4.2), we see that $\Omega$ has at $\omega=\infty$ a simple pole, with residue 1 and zero translation. Thus $\Omega(\omega, \varepsilon)=\omega$, and putting this in (4.2) immediately yields the functional equation (4.1), at least for $w$ off $\Gamma$.

Now we verify the last sentence of the lemma. We merely have to show that both sides of (4.1) are continuous as $w$ tends to a point of $\Gamma_{j}$ (including endpoints) from above. This is true of $\Phi_{0}(w, \varepsilon)$ by definition of a sewing mapping. For $w$ just above $\Gamma_{j}$, we can replace $\Gamma_{j}^{-}$by the path $\Lambda$. The resulting integral is obviously a continuous function of $w$ as $w$ moves onto $\Gamma_{j}$ from above.

LEMMA 4.2. If we restrict $w$ to any region $E$ whose closure does not meet $\Gamma^{-}$ except perhaps at endpoints of arcs $\Gamma_{j}$, we have, uniformly,

$$
\Phi_{0}(w, \varepsilon)=w-\frac{\varepsilon}{2 \pi i} \int_{\Gamma} \frac{\chi(x, 0)}{x-w} d x+o(\varepsilon) .
$$

Proof. The functional equation (4.1) is valid in $E$, and in the course of proving Lemma 4.1 we saw that the integral on the right-hand side of (4.1) is bounded, independently of $\varepsilon$, thus $\Phi_{0}(w, \varepsilon)-w$ tends uniformly to zero in $E$. It follows that $\Phi_{0}^{\prime}(w, \varepsilon)$ tends uniformly to 1 in any region whose closure does not meet either edge of $\Gamma$.

Consider

$$
\int_{\Gamma_{j}^{-}} \frac{\chi_{j}(x, \varepsilon) \Phi_{0}^{\prime}(x, \varepsilon)}{\Phi_{0}(x, \varepsilon)-\Phi_{0}(w, \varepsilon)} d x
$$

and let $\delta>0$ be preassigned. Again we move $\Gamma_{j}^{-}$to a path $\Lambda$ lying below $\Gamma_{j}^{-}$, but this time $\Lambda=\Lambda(\delta)$, and the quantities $s, d$ introduced in the proof of Lemma 4.1 are replaced by $s(\delta), d(\delta)$, chosen so that

$$
\left|\Phi_{0}^{\prime}(x, \varepsilon) /\left(\Phi_{0}(x, \varepsilon)-\Phi_{0}(w, \varepsilon)\right)-1 /(x-w)\right|<\delta
$$

for $w$ in $E$, $x$ on $\Lambda_{3}(\delta)$, and $\varepsilon<\varepsilon_{0}(\delta)$. Then, corresponding to (4.5),

$$
\begin{gathered}
\left|\frac{\varepsilon}{2 \pi i} \int_{\Lambda_{3}(\delta)} \chi_{j}(x, \varepsilon)\left\{\frac{\Phi_{0}^{\prime}(x, \varepsilon)}{\Phi_{0}(x, \varepsilon)-\Phi_{0}(w, \varepsilon)}-\frac{1}{x-w}\right\} d x\right| \\
<M \varepsilon \delta \int_{\Lambda_{3}(\delta)}|d x|=O(\delta \varepsilon)
\end{gathered}
$$


since we can certainly arrange that $\Lambda_{3}(\delta)$ has bounded length, while for $k=1,2$ we have corresponding to (4.6) the estimate

$$
\begin{aligned}
\left|\frac{1}{2 \pi i} \int_{\Lambda_{k}(\delta)} \frac{\chi_{j}(x, \varepsilon) \Phi_{0}^{\prime}(x, \varepsilon)}{\Phi_{0}(x, \varepsilon)-\Phi_{0}(w, \varepsilon)} d x\right| & <\frac{4 A}{C} \int_{0}^{s(\delta)}{ }^{\mu-1} d r \\
& =o\left((s(\delta))^{\mu}\right) .
\end{aligned}
$$

This shows that for $w$ in $E$,

$$
\Phi(w, \varepsilon)=w-\frac{\varepsilon}{2 \pi i} \int_{\Gamma^{-}} \frac{\chi_{j}(x, \varepsilon)}{x-w} d x+\varepsilon e(\delta),
$$

where $e(\delta)$ denotes a quantity tending to zero with $\delta$, which in turn can be made a function of $\varepsilon$ which tends to zero with $\varepsilon$. By continuity we can replace $\chi_{j}(x, \varepsilon)$ by $\chi_{j}(x, 0)$ with a further error $o(1)$. Since $\chi_{j}$ is defined on $\Gamma$, without a jump, we can regard $\Gamma$ rather than $\Gamma^{-}$as the path of integration. This proves the lemma.

5. The analytic character of $\Phi\left(p, \varepsilon ; p_{0}\right)$. In this section we prove the existence of $\Phi_{\varepsilon}\left(p, 0 ; p_{0}\right)$ (the subscript indicating partial differentiation). We show that $\Phi_{\varepsilon}\left(p, 0 ; p_{0}\right)$ is a reciprocal differential of $M-\Gamma$, with a jump across $\Gamma$ which we compute. (For the definitions of reciprocal and quadratic differentials on a Riemann surface and some of their basic properties, see ([5, pp. 31, 85]).) From this information, we will be able to obtain a global formula for $\Phi_{\varepsilon}\left(p, 0 ; p_{0}\right)$.

LEMMA 5.1. If the normalized sewing mapping $\Phi\left(p, \varepsilon ; p_{0}\right)$ exists for the system $\{M, \Gamma, \varphi\}$, then $\Phi_{\varepsilon}\left(p, 0 ; p_{0}\right)$ exists for all $p$ off $\Gamma^{-}$, and even at the endpoints of arcs $\Gamma_{j}$. If $p$ is restricted to any region whose closure does not meet $\Gamma^{-}$except perhaps at endpoints of arcs $\Gamma_{j}, \Phi\left(p, \varepsilon ; p_{0}\right)$ tends uniformly to $p$ as $\varepsilon \rightarrow 0$.

Proof. Let $H$ be the function $H\left(p, p_{0}\right)$ of $\S 2$, carrying $M$ to $C$. $H$ induces a sewing problem $\{C, \Delta, \psi\}$, let $W$ be the normalized sewing mapping for this system. The $\Delta, \psi$ have all the required smoothness properties. If $\Phi=$ $\Phi\left(p, \varepsilon ; p_{0}\right)$, we have $\Phi=H^{-1} W H$. The continuity and differentiability properties with respect to $\varepsilon$ of $W$, proved in Lemma 4.2, finish the argument.

It follows that $\Phi_{\varepsilon}^{-1}$ exists at $\varepsilon=0$, for

$$
\tilde{p}=\Phi\left(p, \varepsilon ; p_{0}\right)=p+\varepsilon \Phi_{\varepsilon}\left(p, 0 ; p_{0}\right)+o(\varepsilon),
$$

thus

$$
\Phi^{-1}\left(\tilde{p}, \varepsilon ; p_{0}\right)=p=\tilde{p}-\varepsilon \Phi_{\varepsilon}\left(p, 0 ; p_{0}\right)+o(\varepsilon)
$$

so that, observing that $\tilde{p} \rightarrow p$ as $\varepsilon \rightarrow 0$,

$$
\Phi^{-1}\left(p, 0 ; p_{0}\right)=-\Phi_{\varepsilon}\left(p, 0 ; p_{0}\right) \text {. }
$$


LEMMA 5.2. If a sewing mapping exists for the system $\{M, \Gamma, \varphi\}, \Phi_{\varepsilon}\left(p, 0 ; p_{0}\right)$ is an everywhere regular reciprocal differential of $M-\Gamma$.

Proof. By Lemma 3.3, $M(\varepsilon)$ is conformally equivalent to $C$, thus the mapping $H\left(p, \varepsilon ; p_{0}\right)$ sending $M(\varepsilon)$ to $C$, with the same normalization as $H\left(p ; p_{0}\right)$, exists. We have

$$
H\left(\Phi\left(p, \varepsilon ; p_{0}\right), \varepsilon ; p_{0}\right)=H\left(p ; p_{0}\right) .
$$

Setting $\tilde{p}=\Phi\left(p, \varepsilon ; p_{0}\right)$, this equation becomes

$$
H\left(\tilde{p}, \varepsilon ; p_{0}\right)=H\left(\Phi^{-1}\left(\tilde{p}, \varepsilon ; p_{0}\right) ; p_{0}\right) .
$$

Now by the remarks following Lemma 5.1, the right-hand side of (5.2) has a derivative with respect to $\varepsilon$ at $\varepsilon=0$, hence so must the left. $H\left(\tilde{p}, \varepsilon ; p_{0}\right)$ is clearly continuously differentiable with respect to $\tilde{p}$, thus has a total differential at $\varepsilon=0$ (see [7, p. 214] and note that it is easy to extend the argument to complex variables) so we can use the chain rule in the ordinary manner. Now differentiate (5.1) with respect to $\varepsilon$ at $\varepsilon=0$, and observe that $\Phi\left(p, 0 ; p_{0}\right)=p$. We get

$$
H_{\varepsilon}\left(p, 0 ; p_{0}\right)=H^{\prime}\left(p, 0 ; p_{0}\right) \Phi_{\varepsilon}\left(p, 0 ; p_{0}\right) .
$$

Now $H\left(p, \varepsilon ; p_{0}\right)$ is a function of $M$, thus so is its derivative with respect to the parameter $\varepsilon$, which exists for $p$ off $\Gamma^{-} . H^{\prime}\left(p, 0 ; p_{0}\right)$ is a linear differential of $M$. So $\Phi_{\varepsilon}$ is a reciprocal differential of $M-\Gamma$.

LEMMA 5.3. If the sewing mapping exists for the system $\{M, \Gamma, \varphi\}$, then $\Phi_{\varepsilon}\left(p, 0 ; p_{0}\right)$ has a jump from $\Gamma^{-}$to $\Gamma^{+}$equal to $-\chi(q, 0)$.

Proof. Let us recall that, on each $\Gamma_{j}, \varphi_{j}(p, \varepsilon)=p+\varepsilon \chi_{j}(p, \varepsilon)$, which in terms of the local parameter $\tau$ has a representation $\tau+\varepsilon r_{j}(\tau, \varepsilon)$. Recalling the meaning of $D_{j}$, we can assume $\tau$ maps the entire parametric patch $D_{j}$ onto a plane domain. Working in this plane domain we can imitate the proof of Lemma 4.1 to arrive at the analogue of (4.3), which is

$$
\Omega(\sigma, \varepsilon)=\tau-\frac{\varepsilon}{2 \pi i} \int_{\Gamma_{j}^{-}} \frac{r_{j}(\tau, \varepsilon) \Phi_{0}^{\prime}(\tau, \varepsilon)}{\Phi_{0}(t, \varepsilon)-\Phi_{0}(\tau, \varepsilon)} d t
$$

where for convenience in notation we let $\Phi_{0}(\tau, \varepsilon)$ denote $\Phi\left(p, \varepsilon ; p_{0}\right)$ referred to the local parameter $\tau$, and $\sigma=\Phi_{0}(\tau, \varepsilon)$. The integral on the right-hand side is uniformly bounded in $D_{j}$, thus (5.4) yields

$$
\Omega(\sigma, \varepsilon)=\tau+o(\varepsilon) .
$$

We cannot completely imitate the proof of Lemma 4.1, because we cannot meaningfully talk about the expansion of $\Omega(\sigma, \varepsilon)$ "about infinity"; indeed $\Omega(\sigma, \varepsilon)$ may not even be defined on all of $M$, it is a local function defined on $\Phi_{0}\left(D_{j}, \varepsilon\right)$. We proceed as follows. In (5.5), we put $\tau=\Phi_{0}^{-1}(\sigma, \varepsilon)$, and apply 
Lemma 5.1 to conclude that $\Omega(\sigma, \varepsilon)$ tends uniformly to $\sigma$ as $\varepsilon \rightarrow 0$ in any region closed away from the set swept out by $\Gamma_{j}(\varepsilon)$ for small $\varepsilon$. This implies that $\Omega^{\prime}(\sigma, \varepsilon)$ tends uniformly to 1 in any such region, and thus about any $\sigma$ there is a local inverse $\Omega^{-1}$. Now apply $\Omega^{-1}$ to both sides of (5.4), to get

$$
\Phi_{0}(\tau, \varepsilon)=\Omega^{-1}\left(\tau-\frac{\varepsilon}{2 \pi i} \int_{\Gamma_{j}^{-}} \frac{r_{j}(t, \varepsilon) \Phi_{0}^{\prime}(t, \varepsilon)}{\Phi_{0}(t, \varepsilon)-\Phi_{0}(\tau, \varepsilon)} d t\right) .
$$

Now differentiate both sides with respect to $\varepsilon$, at $\varepsilon=0$. Since $\Omega^{-}(\sigma, 0)$ has derivative 1 , we get

$$
\Phi_{\varepsilon}\left(p, 0 ; p_{0}\right)=-\frac{\partial}{\partial \varepsilon}\left\{\frac{\varepsilon}{2 \pi i} \int_{\Gamma_{j}^{-}} \frac{r_{j}(t, \varepsilon) \Phi_{0}^{\prime}(t, \varepsilon)}{\Phi(t, \varepsilon)-\Phi(\tau, \varepsilon)} d t\right\} / \varepsilon=0 .
$$

The integral appearing on the right-hand side of (5.6) can be studied by precisely the same method used in Lemma 4.2. We get, in terms of the specific parameters $\tau, t$,

$$
\Phi_{\varepsilon}\left(p, 0 ; p_{0}\right)=-\frac{1}{2 \pi i} \int_{\Gamma_{j}^{-}} \frac{r_{j}(t, 0)}{t-\tau} d t
$$

which, by the theory of Cauchy integrals $[2, \mathrm{p} .42]$ has the jump $-r_{j}(t, 0)$ across $\Gamma_{j}$. But this is just the representation of $-\chi_{j}(q, 0)$ in terms of a particular uniformizer.

We now obtain a global representation for $\Phi_{\varepsilon}\left(p, 0 ; p_{0}\right)$. We introduce the variation kernel $n(p, q)$ given by $[5,7.10 .2]$ which on the Riemann surface $M$ can be written as

$$
n(p, q)=\left(\frac{f\left(p ; p_{0}\right)}{f\left(q ; p_{0}\right)}\right)^{3} \frac{\left(f^{\prime}\left(q ; p_{0}\right)\right)^{2}}{f^{\prime}\left(p ; p_{0}\right)\left[f\left(p ; p_{0}\right)-f\left(q ; p_{0}\right)\right]}
$$

and to simplify notation let us now suppress the parameter $p_{0} . n(p, q)$ transforms as a reciprocal differential in $p$ and a quadratic differential in $q$, and is everywhere regular except if $p=q$, where $n(p, q)$ has a simple pole with residue 1 if we use the same uniformizer for $p$ and $q$.

Now since $D_{j}$ is a parametric patch, we are free to regard $\chi_{j}(q, 0)$ as a reciprocal differential of $D_{j}$, thus the expression

$$
I_{j}(p)=\frac{1}{2 \pi i} \int_{\Gamma_{j}} \chi_{j}(q, 0) n(p, q) d q
$$

is meaningful, and is a reciprocal differential of $M-\Gamma$ if $f$ does not have its pole on the closure of $\Gamma_{j}$. If we restrict the variables $p, q$ to $D_{j}$ and use the same uniformizer for both, $I_{j}(p)$ becomes an ordinary integral with a Cauchy-type kernel. As $p$ crosses $\Gamma_{j}$ from $\Gamma_{j}^{-}$to $\Gamma_{j}^{+}, I_{j}(p)$ has a jump $\chi_{j}(p, 0)$. The argument of Lemma 4.1 shows that $I_{j}(p)$ is bounded in the neighborhood 
of the endpoints of $\Gamma_{j}$. If $f$ has its pole on the closure of $\Gamma_{j}$, we just let $f(p)=1 / F(p)$, and find that

$$
n(p, q)=\left(F^{\prime}(q)\right)^{2} / F^{\prime}(p)[F(p)-F(q)],
$$

showing that $n(p, q)$ retains all relevant properties at the simple pole of $f$.

Now consider the functional

$$
\Phi_{\varepsilon}\left(p, 0 ; p_{0}\right)+\frac{1}{2 \pi i} \int_{\Gamma} \chi(q, 0) n(p, q) d q .
$$

Because the jumps across $\Gamma$ cancel, (5.9) is a reciprocal differential on $M$, with the endpoints of the $\Gamma_{j}$ as the only possible singularities. By a remark in the preceding paragraph, however, these singularities are removable, so (5.9) is an everywhere finite reciprocal differential of $M$. Using [5, p. 86] and the easily verified fact that conformal correspondence of Riemann surfaces carries reciprocal differentials to each other,

$$
\Phi_{\varepsilon}\left(p, 0 ; p_{0}\right)+\frac{1}{2 \pi i} \int_{\Gamma} \chi(q, 0) n(p, q) d q=\frac{A+B f(p)+C(f(p))^{2}}{f^{\prime}(p)}
$$

for certain constants $A, B, C$. To evaluate these constants we compare expansions of both sides in terms of the special uniformizer $\tau$ about $p_{0}$. The left-hand side of (5.10) is $O\left(\tau^{3}\right)$. Using (5.7) we see without difficulty that $A=B=C=0$. Thus

$$
\Phi_{\varepsilon}\left(p, 0 ; p_{0}\right)=-\frac{1}{2 \pi i} \int_{\Gamma} \chi(q, 0) n(p, q) d q .
$$

6. Variational formulae for the conformal mapping. In this section and the next, we simplify notation by taking $f\left(p, \varepsilon ; p_{0}\right)=f(p, \varepsilon)$, and $f(p, 0)=f(p)$.

We obtain the first variation of $f$ by differentiating (3.1), using (5.11) and the fact that $\Phi\left(p, 0 ; p_{0}\right)=p$, to obtain

$$
\begin{aligned}
f_{\varepsilon}(p, 0) & =\frac{-f^{\prime}(p)}{2 \pi i} \int_{\Gamma} \chi(q, 0) n(p, q) d q \\
& =-\frac{(f(p))^{3}}{2 \pi i} \int_{\Gamma} \frac{\chi(q, 0)\left(f^{\prime}(q)\right)^{2}}{(f(q))^{3}[f(p)-f(q)]} d q,
\end{aligned}
$$

this formula valid for all $p$ off $\Gamma^{-}$, and (6.1) is valid also at endpoints of the arcs $\Gamma_{j}$.

It is possible, under certain additional hypotheses, to give a simpler variational formula, (6.2) below, in which the powers of $f(p), f(q)$ are reduced. Note that (6.2) more closely resembles a formula given by Schaeffer and Spencer [4, p. 173] for the extremal schlicht functions mapping the unit disc to the plane slit along a single analytic arc. 
THEOREM 6.1. Suppose the sewing variation is such that $f(p, \varepsilon)$ has its pole at a fixed vertex of $\Gamma$, as $\varepsilon$ varies. Suppose further that (2.1) holds with $\mu=1$.

Then

$$
f_{\varepsilon}(p, \varepsilon)=f(p)-\frac{\varepsilon}{2 \pi i}(f(p))^{2} \int_{\Gamma} \frac{\chi(q, 0)\left(f^{\prime}(q)\right)^{2} d q}{(f(q))^{2}[f(p)-f(q)]}+o(\varepsilon),
$$

the $o(\varepsilon)$ being uniform as $p$ ranges through any set $E$ whose closure does not meet $\Gamma^{-}$save at endpoints of arcs $\Gamma_{j}$.

One has in addition an orthogonality relation

$$
\int_{\Gamma} \frac{\chi(q)\left(f^{\prime}(q)\right)^{2}}{(f(q))^{3}} d q=0
$$

Proof. We retrace the steps leading from (5.7) to (6.1), but replacing $n(p, q)$ by the function

$$
n^{*}(p, q)=f(q) n(p, q) / f(p) .
$$

Consider the integral

$$
I_{j}^{*}(p)=\frac{1}{2 \pi i} \int_{\Gamma_{j}} \chi_{j}(q, 0) n^{*}(p, q) d q .
$$

The new difficulty is that (5.8) is not true, so we must check that $I_{j}^{*}(p)$ remains bounded near the pole of $f$. If we write $f=1 / F$, we have

$$
I_{j}^{*}(p)=\frac{1}{2 \pi i} \frac{F(p)}{F^{\prime}(p)} \int_{\Gamma_{j}} \chi_{j}(q, 0) \frac{\left(F^{\prime}(q)\right)^{2}}{F(q)} \frac{d q}{F(p)-F(q)} .
$$

Because of the strengthened form of $(2.1), \chi_{j}(q, 0) / F(q)$ is bounded in $D_{j}$, so the integral on the right-hand side of (6.4) can be studied by the method of Lemma 4.1. $I_{j}^{*}(p)$ itself tends to zero as $p$ tends to the pole of $f$. Thus it is still true that

$$
\Phi_{\varepsilon}\left(p, 0 ; p_{0}\right)-\frac{1}{2 \pi i} \int_{\Gamma} \chi(q, 0) n^{*}(p, q) d q
$$

is an everywhere finite reciprocal differential of $M$, and we have (5.10) with $n(p, q)$ replaced by $n^{*}(p, q)$. As before we compare expansions in terms of the special parameter $\tau$ about $p_{0}$, this time obtaining

$$
A=B=0, \quad C=-\frac{1}{2 \pi i} \int_{\Gamma} \chi(q, 0)\left(\frac{f^{\prime}(q)}{f(q)}\right)^{3} d q .
$$

We have thus arrived at the relation

$$
\Phi_{\varepsilon}\left(p, 0 ; p_{0}\right)+\frac{1}{2 \pi i} \int_{\Gamma} \chi(q, 0) n^{*}(p, q) d q=\frac{C(f(p))^{2}}{f^{\prime}(p)}
$$


with $C$ given by (6.5). Now we evaluate $C$ in a different way by comparing expansions in terms of a local parameter $\sigma$ about the pole, call it $p_{1}$, of $f$, with $\sigma\left(p_{1}\right)=0$. Since $p_{1}$ is fixed, we have $f(p, \varepsilon)=a(\varepsilon) / \sigma+\ldots$ We see from (3.1) that

$$
\Phi_{\varepsilon}\left(p, 0 ; p_{0}\right) \sim \sigma a^{\prime}(0) / a(0),
$$

which vanishes at $p_{1}$, and we have already noticed from (6.4) that the integral in (6.6) vanishes at $p=p_{1}$. On the other hand the right-hand side of (6.6) tends to a nonzero multiple of $C$ as $\sigma$ tends to zero.

This concludes the proof.

7. Regular behavior of $f\left(p, \varepsilon ; p_{0}\right)$ as a function of $\varepsilon$. As before, let us suppress $p_{0}$ in our notation, taking $f(p, \varepsilon)=f\left(p, \varepsilon ; p_{0}\right)$. We wish to show that under suitable conditions, $f(p ; c)$ is analytic in the parameter $c$. This will be accomplished by showing that the first variation $f_{\varepsilon}(p ; c)$ is a regular function of $c$. The idea is to regard the sewing process which leads from $M$ to $M(\varepsilon)$ as stopping momentarily at $\varepsilon=c$, then starting anew to lead from $M(c)$ to $M(\varepsilon)$. We assume that $c$ and $\varepsilon$ lie in the interval $\left[-\varepsilon_{0}, \varepsilon_{0}\right]$ where our various hypotheses are satisfied.

The sewing process which changed $M$ to $M(\varepsilon)$ was described in terms of a re-identification of edges of $\Gamma_{j}$ on $M$, so that $p$ on $\Gamma_{j}^{-}$was identified with $p+\varepsilon \chi_{j}(p, \varepsilon)$ on $\Gamma_{j}^{+}$. If now we regard $\Gamma_{j}(c)$ as the arcs on $M(c)$, which are just the $\Gamma_{j}$ with the opposite edges identified by $\varphi(p, c)=p+c \chi_{j}(p, c)$, the new re-identification of edges of arcs which changes $M(c)$ to $M(\varepsilon)$ can be described as follows; $p$ on $\Gamma_{j}^{-}(c)$ is to be identified with a point

$$
\varphi(p, \tilde{\varepsilon} ; c)=p+\tilde{\varepsilon} \chi_{j}(p, \tilde{\varepsilon} ; c)
$$

on $\Gamma_{j}^{+}(c)$, with $\tilde{\varepsilon}=\varepsilon-c$. We take

$$
\chi_{j}\left(p, \varepsilon ; p_{0}\right)=\chi_{j}(p, \varepsilon), \quad \varphi\left(p, \varepsilon ; p_{0}\right)=\varphi(p, \varepsilon) .
$$

Thus we have a family of sewing problems $\{M(c), \Gamma(c), \varphi(, ; c)\}$. Because all these surfaces are conformally equivalent to $C$, the sewing mappings $\Phi(p, \varepsilon$; $\left.p_{0} ; c\right)$ exist, by Lemma 3.3. The theory developed in preceding sections is still just as valid for the new sewing problem, and we have, by (6.1),

$$
f_{\varepsilon}(p ; c)=-\frac{(f(p ; c))^{3}}{2 \pi i} \int_{\Gamma} \frac{\chi(q, 0 ; c)\left(f^{\prime}(q ; c)\right)^{2}}{(f(q ; c))^{3}[f(p ; c)-f(q ; c)]} d q
$$

with a similar analogue to (6.2), (6.3) for functions $f$ having their pole at a fixed vertex of $\Gamma$. We should remark that because of the conformal equivalence of $M$ and $M(c)$, all the regularity conditions imposed in $\$ 2$ on the arcs $\Gamma_{j}$, etc., carry over to the new sewing problem $\{M(c), \Gamma(c), \varphi(, ; c)\}$. (In (7.1), primes denote differentiation with respect to the first argument.) 
LEMMA 7.1. Let (7.1) be valid, and suppose $\chi(q, 0 ; c)$ is of class $C^{n}$ as a function of $c$. Then $f(p ; c)$ is of class $C^{n}$ in $c$ for $p$ off $\Gamma$. If $\chi(q, 0 ; c)$ is analytic in $c$, so is $f(p ; c)$, for $p$ off $\Gamma$.

Proof. The existence of the first variation $f_{\varepsilon}(p ; c)$ shows $f(p ; c)$ is continuous in $c$, and thus $f^{\prime}(p ; c)$ is continuous in $c$. Then (7.1) shows $f_{\varepsilon}(p ; c)$ is continuous in $c$. Now we differentiate (7.1) with respect to $c$, using continuity of $f_{\varepsilon}(p ; c)$, to conclude $f_{\varepsilon \varepsilon}(p ; c)$ is continuous in $c$. We iterate this procedure until we run out of derivatives of $\chi$. This concludes the proof.

Now we find the proper way to describe the sewing relative to $M(c)$, and we shall discover in doing so any further conditions we need on $\chi(q, \varepsilon)$ to be able to apply Lemma 7.1.

Let $\tau$ be a local parameter on $M$. Then on $M(c)$ we have local parameters $\sigma(p)$, with $\sigma(p)=\tau(p)$ for $p$ off $\Gamma$, and for a point $p^{*}$ lying over $p$ and $\varphi_{j}(p, \varepsilon)$ on $\Gamma_{j}$, that is points of $\Gamma_{j}(c)$,

$$
\sigma\left(p^{*}\right)= \begin{cases}\tau, & p^{+} \in \Gamma_{j}^{-}(c), \\ \tau+\varepsilon r_{j}(\tau, c), & p^{*} \in \Gamma_{j}^{+}(c) .\end{cases}
$$

Now the sewing leading from $M(c)$ to $M(\varepsilon)$ is described in terms of $\sigma$ by, say,

$$
\sigma \rightarrow \sigma+\tilde{\varepsilon} s_{j}(\sigma, \tilde{\varepsilon}), \quad \tilde{\varepsilon}=\varepsilon-c .
$$

On the lower edge of $\Gamma_{j}(c), \sigma=\tau$, while in describing the point of $\Gamma_{j}^{+}(c)$ which is to be sewn onto this point on the lower edge, we must express $r_{j}(\tau, \varepsilon)$ in terms of $\sigma$. We now complete $s_{j}$. We have

$$
\begin{aligned}
\tau+\varepsilon r_{j}(\tau, \varepsilon)= & \tau+(\varepsilon-c)\left[r_{j}(\tau, c)+\left(r_{j}(\tau, \varepsilon)-r_{j}(\tau, c)\right)\right] \\
& +c r_{j}(\tau, c)+c\left[r_{j}(\tau, \varepsilon)-r_{j}(\tau, c)\right] \\
= & \sigma+\tilde{\varepsilon}\left[r_{j}(\tau, c)+\left(r_{j}(\tau, \varepsilon)-r_{j}(\tau, c)\right)\right] \\
& +c \frac{r_{j}(\tau, \varepsilon)-r_{j}(\tau, c)}{\tilde{\varepsilon}} \tilde{\varepsilon}
\end{aligned}
$$

so that we have

$$
\begin{aligned}
s_{j}(\sigma, \tilde{\varepsilon})= & r_{j}(\tau, c)+r_{j}(\tau, c+\tilde{\varepsilon})-r_{j}(\tau, c) \\
& +c \frac{r_{j}(\tau, c+\tilde{\varepsilon})-r_{j}(\tau, c)}{\tilde{\varepsilon}}
\end{aligned}
$$

with $\tau=\tau(\sigma)$, determined from

$$
\sigma=\tau+c r_{j}(\tau, c)
$$

We observe that, for sufficiently small $c$ so that $d \sigma / d \tau$ is bounded away from zero, we can indeed solve $\left(7.2^{\prime}\right)$ for $\sigma$ as an analytic function of $\tau$, and 
substitute in (7.2). $\Gamma$ is still smooth in terms of $\sigma$, and we still have domains $D_{j}(c)$ on $M(c)$ to work with, satisfying the condition of $\S 2 . s_{j}(\sigma, \varepsilon)$ satisfies the analytic properties of $\S 2$, save perhaps for the Hölder-condition at endpoints of the $\Gamma_{j}(c)$, and to meet this difficulty we impose a new condition on $\Gamma_{j}$; we require

$$
\left|\frac{r_{j}\left(\tau, \varepsilon_{2}\right)-r_{j}\left(\tau, \varepsilon_{1}\right)}{\varepsilon_{2}-\varepsilon_{1}}\right|<A\left|\tau-\tau\left(a_{j, k}\right)\right|^{\mu}, \quad k=1,2 ; \mu>0,
$$

for a fixed $A$ independent of $\varepsilon_{1}, \varepsilon_{2}$. Since $\tau-\tau\left(a_{j, k}\right)$ and $\sigma-\sigma\left(a_{j}, k\right)$ are of the same order of magnitude, this ensures that $s_{j}(\sigma, \tilde{\varepsilon})$ satisfies the uniform Hölder-condition at endpoints of the $\Gamma_{j}(c)$.

With (7.3) assumed, the considerations of $\S \S 4$ and 5 apply to the sewing problem $\{M(c), \Gamma(c), \varphi(, ; c)\}$, and (7.1) holds. To compute $\chi(q, 0 ; c)$, let $\tilde{\varepsilon}$ tend to zero in (7.2), then pass from local parameters to point functions on $M(c)$. We have, putting $\chi(q, \varepsilon ; 0)=\chi(q, \varepsilon)$ for simplicity.

$$
\chi(q, 0 ; c)=\chi(g(q, c), c)+c \chi_{\varepsilon}(g(q, c), c)
$$

where $g(q, c)$ is the point function which writes itself in terms of the local parameters $\sigma$ and $\tau$ as

$$
g: \sigma=\tau+\operatorname{cr}(\tau, c) .
$$

It is easy to deduce differentiability properties of $g$ and hence $\chi$ from those of $r$. We summarize our discussion by stating a theorem, which has already been almost completely proved.

THEOREM 7.1. Let $\chi(\tau, \varepsilon)$ have $n$ continuous derivatives with respect to $\varepsilon$, and satisfy, in addition to the requirements of $\$ 2$, the condition (7.3) in terms of the representative $r(\tau)$.

Then $f\left(p, c ; p_{0}\right)$ has $n$ continuous derivatives with respect to $c$, for $p$ off $\Gamma$ or a vertex of $\Gamma$.

If $\chi(\tau, \varepsilon)$ is analytic in its dependence on $\varepsilon, f\left(p, c ; p_{0}\right)$ is analytic in $c$, for $p$ off $\Gamma$ or a vertex of $\Gamma$.

If $p$ is a vertex of $\Gamma$ at which $f\left(p, 0 ; p_{0}\right)$ has its pole, we are to interpret the above as meaning that $1 / f\left(p, \varepsilon ; p_{0}\right)$ varies smoothly with $\varepsilon$.

Proof. The only parts of this theorem that perhaps remain to be verified are the statements concerning the behavior of $f\left(v, p_{0} ; c\right)$ for $v$ an endpoint of an arc. From Lemma 5.1 adapted to our new context, $\Phi_{\varepsilon}\left(p, 0 ; p_{0} ; c\right)$ exists at the endpoints of the $\Gamma_{j}$, in fact at every point of $\Gamma_{j}^{+}(c)$. The analogue on $M(c)$ of $(5.11)$ holds on $\Gamma(c)$ since both sides are continuous as $p$ moves onto $\Gamma(c)$ from above. This shows that $\Phi_{\varepsilon}\left(v, 0 ; p_{0} ; c\right)$ is smooth in its dependence on $c$. (3.1) then shows $f\left(v, \varepsilon ; p_{0}\right)$ varies smoothly with $c$, provided $v$ is not a pole of 
$f$, and if $v$ is a pole of $f$ we find that $1 / f\left(v, \varepsilon ; p_{0}\right)$ varies analytically with $\varepsilon$ by using uniformizers at $\infty$ in the image space.

\section{REFERENCES}

1. R. Courant, Dirichlet's principle, conformal mapping, and minimal surfaces, Interscience, New York, 1950. MR 12, 90.

2. N. I. Mushelisvili, Singular integral equations. Boundary problems of function theory and their application to mathematical physics, OGIZ, Moscow, 1946; 2nd ed., Fizmatgiz, Moscow, 1962; English transl., Noordhoff, Groningen, 1953. MR 8, 586; 15, 434.

3. Zeev Nehari, Conformal mapping, International Series, McGraw-Hill, New York, 1952. MR 13, 640 .

4. A. C. Schaeffer and D. C. Spencer, Coefficient regions for schlicht functions, Amer. Math. Soc. Colloq. Publ., vol. 35, Amer. Math. Soc., Providence, R. I., 1950. MR 12, 326.

5. M. M. Schiffer and D. C. Spencer, Functionals of finite Riemann surfaces, Princeton Univ. Press, Princeton, N. J., 1954. MR 16, 461.

6. George Springer, Introduction to Riemann surfaces, Addison-Wesley, Reading, Mass., 1957. MR 19, 1169.

7. A. E. Taylor, Advanced calculus, Blaisdell, Waltham, Mass., 1955.

Department of Mathematics, Michigan State University, East lansing, Michigan 48824 